\title{
A local minimum theorem and critical nonlinearities
}

\author{
G. Bonanno, G. D'Aguì and D. O'Regan
}

\begin{abstract}
In this paper the existence of two positive solutions for a Dirichlet problem having a critical growth, and depending on a real parameter, is established. The approach is based on methods which are totally variational, unlike the fundamental result of Ambrosetti, Brezis and Cerami where a clever combination of topological and variational methods is used in order to obtain the same conclusion. In addition, a numerical estimate of real parameters, for which the two solutions are obtained, is provided. Our main tool is a local minimum theorem.
\end{abstract}

\section{Introduction}

In the classical and seminal paper of Brezis and Nirenberg (see [9]), Dirichlet problems with a critical growth are investigated. This study presents several difficulties since the critical growth of the nonlinearity leads to the fact that the associated functional is not sufficiently regular. Indeed, the Palais-Smale condition, as well as the weak lower semi-continuity of the associated functional may fail because of the fact that the embedding $H_{0}^{1}(\Omega)$ in $L^{\frac{2 N}{N-2}}(\Omega)$ is not compact. To be precise, consider the problem

$$
\left\{\begin{array}{l}
-\Delta u=u^{\frac{N+2}{N-2}}+g(u) \text { in } \Omega \\
u>0 \text { in } \Omega \\
\left.u\right|_{\partial \Omega}=0
\end{array}\right.
$$

Key Words: Critical growth, nonlinear differential problem, variational methods, PalaisSmale condition, local minimum.

2010 Mathematics Subject Classification: 35J60, 35J20, 58E30, 49J52, 49J50.

Received: 20.04.2015

Revised: 10.05 .2015

Accepted: 18.05.2015 
where $\Omega$ is a non-empty bounded open subset of the Euclidean space $\left(\mathbb{R}^{N},|\cdot|\right)$, $N \geq 3$, with boundary of class $C^{1}$, and $g: \mathbb{R} \rightarrow \mathbb{R}$ is a non-zero continuous lower-order perturbation of $u^{\frac{N+2}{N-2}}$ in the sense that $0 \leq g(u) \leq \mu u^{s}$ for all $u \in \mathbb{R}$, for some $\mu>0$ and $0<s<\frac{N+2}{N-2}$, for which a typical example is $g(u)=\mu|u|^{s}$. Brezis and Nirenberg in [9] (note the embedding $H_{0}^{1}(\Omega) \hookrightarrow$ $L^{\frac{2 N}{N-2}}(\Omega)$ is not compact) starting from the well-known nonexistence result of Pohozaev [11] for which $(D)$ has no solutions when $g \equiv 0$ proved that $(D)$ admits at least one solution provided that $g$ is linear or superlinear at zero. In particular, they established that, when $g(u)=\mu|u|^{s}$, problem $(D)$ admits a solution for suitable values of $\mu$, provided that $1 \leq s<\frac{N+2}{N-2}$. Hence, a lower-order perturbation, which is linear or super-linear at zero, can reverse the situation highlighted by Pohozaev.

Subsequently, Ambrosetti, Brezis and Cerami in the seminal paper [1] proved that even if the lower-order perturbation is sublinear at zero, that is, $0<s<1$, the problem admits again solutions for suitable value of $\mu$ that, indeed in this case, are at least two. In particular, they proved the following result.

Theorem 1.1. (See [1, Theorem 2.3]). Fix $0<s<1$. Then, there is $\Lambda>0$ such that for each $\mu \in] 0, \Lambda[$ problem

$$
\left\{\begin{array}{l}
-\Delta u=u^{\frac{N+2}{N-2}}+\mu u^{s} \quad \text { in } \Omega, \\
u>0 \text { in } \Omega, \\
\left.u\right|_{\partial \Omega}=0
\end{array}\right.
$$

admits at least two weak solutions.

Moreover, they also proved that if $\mu>\Lambda$, the previous problem admits no solution (see [1, Theorem 2.1]). Their proof is a clever combination of topological and variational methods. Precisely, they determine the existence of a first solution by using the method of sub- and super-solutions and then, through a deep reasoning, prove that this solution is the minimum of a suitable functional and apply the mountain pass theorem so ensuring the existence of a second solution. However, in their proof, no numerical estimate of $\Lambda$ is provided.

The purpose of this paper is to give an alternative proof of Theorem 1.1 which is exclusively variational. Moreover, thanks to this novel variational proof, a precise numerical estimate of $\Lambda$ is provided and we can solve specific problems in which the result of Ambrosetti, Brezis and Cerami cannot be applied (see Example 4.1 and Remark 4.4). Our main tool is a local minimum theorem established in [3] (see Theorem 3.1). Here, we apply one of its consequence given in [4] (see Theorem 3.3). For completeness and clarity we recall their proofs in Section 3. From Theorem 3.3 we establish an existence result 
for one positive weak solution for the problem which is a local minimum of the associated energy functional and where a numerical estimate of $\Lambda$ is provided (see Theorem 4.1). It is worth noticing again that the solution ensured by Theorem 4.1 is directly obtained as a local minimum, contrary to $[1$, Theorem 2.1], where the solution is ensured by the method of sub- and super-solutions. Finally, Theorem 4.2 ensures the existence of a second solution, by starting from the fact that the first solution is a local minimum also for a modified suitable functional and then applying the mountain pass theorem exactly as in $[1]$.

The paper is arranged as follows. Section 2 presents basic definitions and preliminaries. In particular, a consequence of the Ekeland variational principle built in a non-smooth framework (see Lemma 2.1) is recalled. In Section 3, the local minimum theorem is reported (Theorem 3.1) and special cases are pointed out (Theorems 3.2 and 3.3), while in Section 4 a Dirichlet problem with critical nonlinearities is investigated. To be precise, a type of PalaisSmale condition for the energy functional associated to an elliptic Dirichlet problem is proved (Lemma 4.1), existence results of one and two solutions are established (see Theorems 4.1 and 4.2) and an example for which ([1, Theorem 2.1]) cannot be applied, is pointed out (see Example 4.1).

\section{Preliminaries}

Let $(X,\|\cdot\|)$ be a real Banach space. We denote the dual space of $X$ by $X^{*}$, while $\left\langle\cdot, \cdot>\right.$ stands for the duality pairing between $X^{*}$ and $X$. A function $I: X \rightarrow \mathbb{R}$ is called locally Lipschitz when, to every $u \in X$, there corresponds a neighbourhood $U$ of $u$ and a constant $L \geq 0$ such that

$$
|I(v)-I(w)| \leq L\|v-w\| \quad \text { for all } v, w \in U
$$

If $u, v \in X$, the symbol $I^{\circ}(u ; v)$ indicates the generalized directional derivative of $I$ at point $u$ along direction $v$, namely

$$
I^{\circ}(u ; v):=\limsup _{w \rightarrow u, t \rightarrow 0^{+}} \frac{I(w+t v)-I(w)}{t} .
$$

The generalized gradient of the function $I$ at $u$, denoted by $\partial I(u)$, is the set

$$
\partial I(u):=\left\{u^{*} \in X^{*}:<u^{*}, v>\leq I^{\circ}(u ; v) \text { for all } v \in X\right\} .
$$

A function $I: X \rightarrow \mathbb{R}$ is called Gâteaux differentiable at $u \in X$ if there is $\varphi \in X^{*}$ (denoted by $\left.I^{\prime}(u)\right)$ such that

$$
\lim _{t \rightarrow 0^{+}} \frac{I(u+t v)-I(u)}{t}=I^{\prime}(u)(v) \quad \forall v \in X .
$$


It is called continuously Gâteaux differentiable if it is Gâteaux differentiable for any $u \in X$ and the function $u \rightarrow I^{\prime}(u)$ is a continuous map from $X$ to its dual $X^{*}$. We recall that if $I$ is continuously Gâteaux differentiable then it is locally Lipschitz and one has $I^{\circ}(u ; v)=I^{\prime}(u)(v)$ for all $u, v \in X$.

Now, let $\Phi, \Psi: X \rightarrow \mathbb{R}$ be two continuously Gâteaux differentiable functionals and put

$$
I=\Phi-\Psi
$$

Fix $r_{1}, r_{2} \in[-\infty,+\infty]$, with $r_{1}<r_{2}$, and we say that the functional $I$ verifies the Palais-Smale condition cut off lower at $r_{1}$ and upper at $r_{2}$ (in short ${ }^{\left[r_{1}\right]}(P S)^{\left[r_{2}\right]}$-condition) if any sequence $\left\{u_{n}\right\}$ such that

$(\alpha)\left\{I\left(u_{n}\right)\right\}$ is bounded,

(及) $\lim _{n \rightarrow+\infty}\left\|I^{\prime}\left(u_{n}\right)\right\|_{X^{*}}=0$,

$(\gamma) r_{1}<\Phi\left(u_{n}\right)<r_{2} \quad \forall n \in \mathbb{N}$,

has a convergent subsequence.

When we fix $r_{2}=-\infty$, that is, $\Phi\left(u_{n}\right)<r_{2} \forall n \in \mathbb{N}$, we denote this type of Palais Smale condition with $(P S)^{\left[r_{2}\right]}$. When, in addition, $r_{2}=+\infty$, it is the classical Palais Smale condition.

Now, we recall the following consequence of the Ekeland variational principle built within a non-smooth framework (see, for example [3, Lemma 3.1]).

Lemma 2.1. Let $X$ be a real Banach space and let $I: X \rightarrow \mathbb{R}$ be a locally Lipschitz function bounded from below. Then, for all minimizing sequence of $I,\left\{u_{n}\right\}_{n \in \mathbb{N}} \subseteq X$, there exists a minimizing sequence of $I,\left\{v_{n}\right\}_{n \in \mathbb{N}} \subseteq X$, such that

$$
\begin{gathered}
I\left(v_{n}\right) \leq I\left(u_{n}\right) \quad \forall n \in \mathbb{N}, \\
I^{\circ}\left(v_{n} ; h\right) \geq-\varepsilon_{n}\|h\| \quad \forall h \in X, \quad \forall n \in \mathbb{N}, \quad \text { where } \varepsilon_{n} \rightarrow 0^{+} .
\end{gathered}
$$

\section{A local minimum theorem and some consequences}

The main result of this section is the following local minimum theorem.

Theorem 3.1. Let $X$ be a real Banach space and let $\Phi, \Psi: X \rightarrow \mathbb{R}$ be two continuously Gâteaux differentiable functions. Put

$$
I=\Phi-\Psi
$$


and assume that there are $x_{0} \in X$ and $r_{1}, r_{2} \in \mathbb{R}$, with $r_{1}<\Phi\left(x_{0}\right)<r_{2}$, such that

$$
\begin{aligned}
\sup _{u \in \Phi^{-1}(] r_{1}, r_{2}[)} \Psi(u) \leq r_{2}-\Phi\left(x_{0}\right)+\Psi\left(x_{0}\right), \\
\sup _{\left.\left.u \in \Phi^{-1}(]-\infty, r_{1}\right]\right)} \Psi(u) \leq r_{1}-\Phi\left(x_{0}\right)+\Psi\left(x_{0}\right) .
\end{aligned}
$$

Moreover, assume that I satisfies ${ }^{\left[r_{1}\right]}(P S)^{\left[r_{2}\right]}$-condition.

Then, there is $u_{0} \in \Phi^{-1}(] r_{1}, r_{2}[)$ such that $I\left(u_{0}\right) \leq I(u)$ for all $u \in \Phi^{-1}(] r_{1}, r_{2}[)$ and $I^{\prime}\left(u_{0}\right)=0$.

Proof. Put

$$
\begin{gathered}
M=r_{2}-\Phi\left(x_{0}\right)+\Psi\left(x_{0}\right), \\
\Psi_{M}(u)= \begin{cases}\Psi(u) & \text { if } \Psi(u)<M \\
M & \text { if } \Psi(u) \geq M,\end{cases} \\
\Phi^{r_{1}}(u)= \begin{cases}\Phi(u) & \text { if } \Phi(u)>r_{1} \\
r_{1} & \text { if } \Phi(u) \leq r_{1},\end{cases} \\
J=\Phi^{r_{1}}-\Psi_{M} .
\end{gathered}
$$

Clearly, $J$ is locally Lipschitz and bounded from below. Now, given a sequence $\left\{u_{n}\right\}_{n \in \mathbb{N}} \subseteq X$ such that $\lim _{n \rightarrow \infty} J\left(u_{n}\right)=\inf _{X} J$, owing to Lemma 2.1 there is a sequence $\left\{v_{n}\right\}_{n \in \mathbb{N}} \subseteq X$ such that $\lim _{n \rightarrow \infty} J\left(v_{n}\right)=\inf _{X} J$ and $J^{\circ}\left(v_{n} ; h\right) \geq$ $-\varepsilon_{n}\|h\|$ for all $h \in X$, for all $n \in \mathbb{N}$, where $\varepsilon_{n} \rightarrow 0^{+}$. If $J\left(x_{0}\right)=\inf _{X} J$ then $x_{0}$ satisfies the conclusion. In fact, if $u \in \Phi^{-1}(] r_{1}, r_{2}[)$ from (3.1) one has $\Psi(u) \leq M$ and $J(u)=I(u)$ for all $u \in \Phi^{-1}(] r_{1}, r_{2}[)$; hence $I\left(x_{0}\right)=$ $J\left(x_{0}\right) \leq J(u)=I(u)$ for all $u \in \Phi^{-1}(] r_{1}, r_{2}[)$. So, we assume $\inf _{X} J<$ $J\left(x_{0}\right)$. Therefore, there is a $\nu \in \mathbb{N}$ such that $J\left(v_{n}\right)<J\left(x_{0}\right)$ for all $n>\nu$. Now, we claim that $r_{1}<\Phi\left(v_{n}\right)<r_{2}$ for all $n>\nu$. On the one hand, one has $\Phi\left(v_{n}\right)-\Psi_{M}\left(v_{n}\right) \leq \Phi^{r_{1}}\left(v_{n}\right)-\Psi_{M}\left(v_{n}\right)<\Phi\left(x_{0}\right)-\Psi\left(x_{0}\right) ; \Phi\left(v_{n}\right)<$ $\Psi_{M}\left(v_{n}\right)+\Phi\left(x_{0}\right)-\Psi\left(x_{0}\right) \leq M+\Phi\left(x_{0}\right)-\Psi\left(x_{0}\right)=r_{2}, \Phi\left(v_{n}\right)<r_{2}$. On the other hand, arguing by contradiction, we assume $\Phi\left(v_{n}\right) \leq r_{1}$. Therefore, one has $r_{1}-\Psi\left(v_{n}\right)=\Phi^{r_{1}}\left(v_{n}\right)-\Psi\left(v_{n}\right)<\Phi\left(x_{0}\right)-\Psi\left(x_{0}\right) ; \Psi\left(v_{n}\right)>r_{1}-\Phi\left(x_{0}\right)+\Psi\left(x_{0}\right)$ and, from (3.2), one has $\Phi\left(v_{n}\right)>r_{1}$, that is a contradiction. Hence, our claim is proved.

Therefore, one has $J\left(v_{n}\right)=I\left(v_{n}\right)$ and $J^{\circ}\left(v_{n} ; h\right)=I^{\prime}\left(v_{n}\right)(h)$ for all $n>\nu$. Hence, $\lim _{n \rightarrow \infty} I\left(v_{n}\right)=\lim _{n \rightarrow \infty} J\left(v_{n}\right)=\inf _{X} J$ and $I^{\prime}\left(v_{n}\right)(h) \geq-\varepsilon_{n}\|h\|$, that is $\lim _{n \rightarrow \infty}\left\|I^{\prime}\left(v_{n}\right)\right\|_{X^{*}}=0$. Since $I$ satisfies ${ }^{\left[r_{1}\right]}(P S)^{\left[r_{2}\right]}$-condition, then $\left\{v_{n}\right\}$ 
admits a subsequence strongly converging to $v^{*} \in X$. Thus, $I\left(v^{*}\right)=\inf _{X} J \leq$ $J(u)=I(u)$ for all $u \in \Phi^{-1}(] r_{1}, r_{2}[)$, that is

$$
I\left(v^{*}\right) \leq I(u)
$$

for all $u \in \Phi^{-1}(] r_{1}, r_{2}[)$.

Since $r_{1}<\Phi\left(v_{n}\right)<r_{2}$ for all $n>\nu$, from the continuity of $\Phi$ we obtain $v^{*} \in \Phi^{-1}\left(\left[r_{1}, r_{2}\right]\right)$.

If $v^{*} \in \Phi^{-1}(] r_{1}, r_{2}[),(3.3)$ immediately ensures the conclusion. If $\Phi\left(v^{*}\right)=r_{1}$, from (3.2) we obtain $I\left(v^{*}\right)=r_{1}-\Psi\left(v^{*}\right) \geq r_{1}-\sup _{\Phi(u) \leq r_{1}} \Psi(u) \geq \Phi\left(x_{0}\right)-$ $\Psi\left(x_{0}\right)=I\left(x_{0}\right)$ and, hence, from (3.3), $I\left(x_{0}\right) \leq I(u)$ for all $u \in \Phi^{-1}(] r_{1}, r_{2}[)$ and the conclusion is achieved. If $\Phi\left(v^{*}\right)=r_{2}$, first we observe that $\Psi\left(v^{*}\right) \leq M$; in fact, taking into account that $I\left(v^{*}\right)=J\left(v^{*}\right)$, one has $r_{2}-\Psi\left(v^{*}\right)=r_{2}-$ $\Psi_{M}\left(v^{*}\right), \Psi\left(v^{*}\right)=\Psi_{M}\left(v^{*}\right) \leq M$. Next, we prove that $I\left(v^{*}\right)=I\left(x_{0}\right)$. In fact, arguing by contradiction and assuming $I\left(v^{*}\right)<I\left(x_{0}\right)$, from $\left(3.1^{*}\right)$ one has $I\left(v^{*}\right)=r_{2}-\Psi\left(v^{*}\right) \geq r_{2}-M=\Phi\left(x_{0}\right)-\Psi\left(x_{0}\right)=I\left(x_{0}\right)$, that is $I\left(v^{*}\right) \geq I\left(x_{0}\right)$ and we have a contradiction. Hence, from (3.3) one has $I\left(x_{0}\right) \leq I(u)$ for all $u \in \Phi^{-1}(] r_{1}, r_{2}[)$ and also in this case the conclusion is achieved.

Now, we point out the following consequence of Theorem 3.1 when the function $I$ depends on a real parameter, that is, it is of the type $\Phi-\lambda \Psi$, with $\lambda>0$. To this end, given $\Phi, \Psi: X \rightarrow \mathbb{R}$, put

$$
\beta\left(r_{1}, r_{2}\right)=\inf _{v \in \Phi^{-1}(] r_{1}, r_{2}[)} \frac{\sup \frac{u \in \Phi^{-1}(] r_{1}, r_{2}[)}{r_{2}-\Phi(v)} \Psi(u)-\Psi(v)}{r_{2}}
$$

for all $r_{1}, r_{2} \in \mathbb{R}$, with $r_{1}<r_{2}$,

$$
\rho_{2}\left(r_{1}, r_{2}\right)=\sup _{v \in \Phi^{-1}(] r_{1}, r_{2}[)} \frac{\Psi(v)-\sup _{\left.\left.u \in \Phi^{-1}(]-\infty, r_{1}\right]\right)} \Psi(u)}{\Phi(v)-r_{1}}
$$

for all $r_{1}, r_{2} \in \mathbb{R}$, with $r_{1}<r_{2}$.

The next result is a consequence of Theorem 3.1.

Theorem 3.2. Let $X$ be a real Banach space and let $\Phi, \Psi: X \rightarrow \mathbb{R}$ be two continuously Gâteaux differentiable functions. Assume that there are $r_{1}$, $r_{2} \in \mathbb{R}$, with $r_{1}<r_{2}$, such that

$$
\beta\left(r_{1}, r_{2}\right)<\rho_{2}\left(r_{1}, r_{2}\right),
$$


where $\beta$ and $\rho_{2}$ are given by (3.4) and (3.5), and for each $\lambda \in] \frac{1}{\rho_{2}\left(r_{1}, r_{2}\right)}, \frac{1}{\beta\left(r_{1}, r_{2}\right)}\left[\right.$ the function $I_{\lambda}=\Phi-\lambda \Psi$ satisfies ${ }^{\left[r_{1}\right]}(P S)^{\left[r_{2}\right]_{-}}$

Then, for each $\lambda \in] \frac{1}{\rho_{2}\left(r_{1}, r_{2}\right)}, \frac{1}{\beta\left(r_{1}, r_{2}\right)}\left[\right.$ there is $u_{0, \lambda} \in \Phi^{-1}(] r_{1}, r_{2}[)$ such that $I_{\lambda}\left(u_{0, \lambda}\right) \leq I_{\lambda}(u)$ for all $u \in \Phi^{-1}(] r_{1}, r_{2}[)$ and $I_{\lambda}^{\prime}\left(u_{0, \lambda}\right)=0$.

Proof. Fix $\lambda$ as in the conclusion. One has $\beta\left(r_{1}, r_{2}\right)<\frac{1}{\lambda}<\rho_{2}\left(r_{1}, r_{2}\right)$, that is

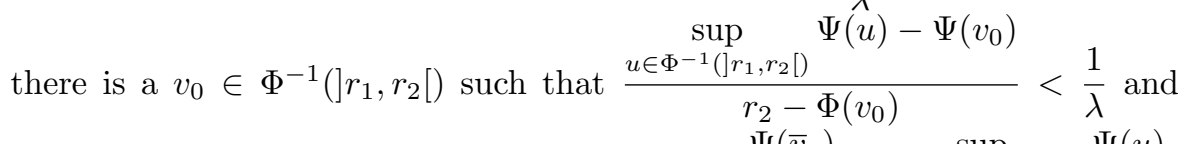
there is a $\bar{v}_{0} \in \Phi^{-1}(] r_{1}, r_{2}[)$ such that $\frac{1}{\lambda}<\frac{\Psi\left(\bar{v}_{0}\right)-\sup _{\left.\left.u \in \Phi^{-1}(]-\infty, r_{1}\right]\right)} \Psi(u)}{\Phi\left(\bar{v}_{0}\right)-r_{1}}$. Therefore, calling $x_{0}$ the point of $\Phi^{-1}(] r_{1}, r_{2}[)$ such that $\Phi\left(x_{0}\right)-\lambda \Psi\left(x_{0}\right)=$ $\min \left\{\Phi\left(v_{0}\right)-\lambda \Psi\left(v_{0}\right), \Phi\left(\bar{v}_{0}\right)-\lambda \Psi\left(\bar{v}_{0}\right)\right\}$, one has

and

$$
\sup _{u \in \Phi^{-1}(] r_{1}, r_{2}[)} \lambda \Psi(u)<r_{2}-\Phi\left(x_{0}\right)+\lambda \Psi\left(x_{0}\right)
$$

$\sup _{\left.\left.u \in \Phi^{-1}(]-\infty, r_{1}\right]\right)} \lambda \Psi(u) \leq r_{1}-\Phi\left(x_{0}\right)+\lambda \Psi\left(x_{0}\right)$. Hence, applying Theorem 3.1 to the function $\Phi-\lambda \Psi$, the conclusion is obtained.

Remark 3.1. We recall that Theorems 3.1 and 3.2 were established in [3] (see [3, Theorem 3.1] and [3, Theorem 5.1]).

Now, we point out a further consequence of the local minimum theorem.

Theorem 3.3. Let $X$ be a real Banach space and let $\Phi, \Psi: X \rightarrow \mathbb{R}$ be two continuously Gâteaux differentiable functionals such that $\inf _{X} \Phi=\Phi(0)=$ $\Psi(0)=0$. Assume that there are $r \in \mathbb{R}$ and $\tilde{u} \in X$, with $0<\Phi(\tilde{u})<r$, such that

$$
\frac{\sup _{u \in \Phi^{-1}(]-\infty, r[)} \Psi(u)}{r}<\frac{\Psi(\tilde{u})}{\Phi(\tilde{u})}
$$

and, for each $\lambda \in] \frac{\Phi(\tilde{u})}{\Psi(\tilde{u})}, \frac{r}{\sup _{u \in \Phi^{-1}(]-\infty, r[)} \Psi(u)}\left[\right.$, the functional $I_{\lambda}=\Phi-\lambda \Psi$ satisfies $(P S)^{[r]}$-condition. 
Then, for each $\lambda \in] \frac{\Phi(\tilde{u})}{\Psi(\tilde{u})}, \frac{r}{\sup _{u \in \Phi^{-1}(]-\infty, r[)} \Psi(u)}\left[\right.$, there is $u_{\lambda} \in \Phi^{-1}(] 0, r[)$ (hence, $u_{\lambda} \neq 0$ ) such that $I_{\lambda}\left(u_{\lambda}\right) \leq I_{\lambda}(u)$ for all $u \in \Phi^{-1}(] 0, r[)$ and $I_{\lambda}^{\prime}\left(u_{\lambda}\right)=$ 0 .

Proof. Our aim is to apply Theorem 3.2. To this end, by choosing $r_{1}=0$ and $r_{2}=r$, we claim that (3.6) holds true. Indeed, from (3.7) one has

$$
\beta(0, r) \leq \frac{\sup _{u \in \Phi^{-1}(]-\infty, r[)} \Psi(u)-\Psi(\tilde{u})}{r-\Phi(\tilde{u})}<\frac{r \frac{\Psi(\tilde{u})}{\Phi(\tilde{u})}-\Psi(\tilde{u})}{r-\Phi(\tilde{u})}=\frac{\Psi(\tilde{u})}{\Phi(\tilde{u})} \leq \rho_{2}(0, r) .
$$

Hence, one has

$$
\beta(0, r)<\rho_{2}(0, r)
$$

and our claim is proved. Moreover, let $\left\{v_{n}\right\} \subseteq \Phi^{-1}(] 0, r[)$ such that $\lim _{n \rightarrow \infty} v_{n}=$ 0 . One has $\beta(0, r) \leq \frac{\sup _{u \in \Phi^{-1}(] 0, r[)} \Psi(u)-\Psi\left(v_{n}\right)}{r-\Phi\left(v_{n}\right)}$ for all $n \in \mathbb{N}$. Therefore, taking into account the continuity of $\Phi$ and $\Psi$, one has

$$
\beta(0, r) \leq \frac{\sup _{u \in \Phi^{-1}(]-\infty, r[)} \Psi(u)}{r}
$$

It follows that $] \frac{\Phi(\tilde{u})}{\Psi(\tilde{u})}, \frac{r}{\sup _{u \in \Phi^{-1}(]-\infty, r[)} \Psi(u)}[\subseteq] \frac{1}{\rho_{2}(0, r)}, \frac{1}{\beta(0, r)}[$.

Finally, we observe that, since $I_{\lambda}$ satisfies $(P S)^{[r]}$-condition, then it satisfies ${ }^{[0]}(P S)^{[r]}$-condition.

Hence, all assumptions of Theorem 3.2 hold and the conclusion is achieved.

Remark 3.2. We recall that Theorem 3.3 was established in [4] (see [4, Theorem 2.3]).

Remark 3.3. Theorem 3.1 has several other consequences, besides Theorems 3.2 and 3.3. In particular, for example, the local minimum theorem established in [7] can be obtained as a special case. Moreover, again from Theorem 3.1, multiple critical points results can be obtained and we refer the reader to [3] for more details. 
Remark 3.4. The proof of Theorem 3.1 is based on a consequence of the classical Ekeland variational principle built in a non-smooth framework (see Lemma 2.1). It is worth noticing that in this case the non-smooth setting is applied in order to obtain novel results in the smooth framework. In particular, we point out that in Theorem 3.1 the sequential weak lower semi-continuity of the functional is not requested, contrary to direct method theorems (see, for example, [13, Theorem 1.2]) and local minimum theorems established in [5] and [12] where it is a fundamental assumption. Elliptic Dirichlet problems with critical exponent, investigated in the next section, as well as nonlinear problems in the whole space (see [2]), are examples for which the sequential weak lower semi-continuity of the associated functional may fail.

\section{Elliptic Dirichlet problems with critical nonlinearities}

In this section we investigate elliptic Dirichlet problems with critical exponent. It is worth noticing that, in this case, the $(P S)$-condition as well as the weak lower semi-continuity of the associated functional may fail. For this reason, classical results, for example direct method theorems (see also Remark 3.4), cannot be used and the local minimum theorem given in Section 3 may be used to obtain nontrivial solutions. Our main result is Theorem 4.1, which ensures the existence of one positive solution by applying Theorem 3.3. Then, as a consequence, we present Theorem 4.2, where two positive solutions are obtained. First, we give the framework of the problem and we establish Lemma 4.1 which is fundamental in the proof of Theorem 4.1.

Consider the Dirichlet problem

$$
\left\{\begin{aligned}
-\Delta u & =\lambda(h(u)+\mu g(u)) \quad \text { in } \quad \Omega \\
\left.u\right|_{\partial \Omega} & =0
\end{aligned}\right.
$$

where $\Omega$ is a non-empty bounded open subset of the Euclidean space $\left(\mathbb{R}^{N},|\cdot|\right)$, $N \geq 3$, with boundary of class $C^{1}, h(t)=t^{2^{*}-1}, g(t)=t^{q-1}$ if $t \geq 0, h(t)=$ $g(t)=0$ if $t<0,2^{*}=\frac{2 N}{N-2}, 1<q<2, \lambda$ and $\mu$ are positive parameters. One has $f(t)=h(t)+\mu g(t) \leq \mu|t|^{q-1}+|t|^{2^{*}-1}$ for all $t \in \mathbb{R}$. As usual, put $X=$ $H_{0}^{1}(\Omega)$ endowed with the norm $\|u\|=\left(\int_{\Omega}|\nabla u(x)|^{2} d x\right)^{\frac{1}{2}}$ and $\Phi(u)=\frac{\|u\|^{2}}{2}$, $\Psi(u)=\int_{\Omega} F(u(x)) d x$ for all $u \in X$, where $F(\xi)=\int_{0}^{\xi} f(t) d t$ for every $\xi \in \mathbb{R}$, that is, $F(\xi)=\int_{0}^{\xi} h(t) d t+\mu \int_{0}^{\xi} g(t) d t=H(\xi)+\mu G(\xi)=\frac{1}{2^{*}}|\xi|^{2^{*}}+\mu \frac{1}{q}|\xi|^{q}$ for all $\xi \geq 0$ and $F(\xi)=0$ for all $\xi<0$. We observe that one has $F(\xi) \geq 0$ for all $\xi \in \mathbb{R}$. 
We recall that

$$
\begin{gathered}
\|u\|_{L^{s}(\Omega)} \leq c_{s}\|u\|, \quad u \in H_{0}^{1}(\Omega), \quad s \in\left[1,2^{*}\right] \\
c_{2^{*}}=\frac{1}{\sqrt{N(N-2) \pi}}\left(\frac{N !}{2 \Gamma(1+N / 2)}\right)^{1 / N}, \\
c_{s} \leq \frac{\operatorname{meas}(\Omega)^{\frac{2^{*}-s}{2^{*}}}}{\sqrt{N(N-2) \pi}}\left(\frac{N !}{2 \Gamma(N / 2+1)}\right)^{1 / N}
\end{gathered}
$$

and that the embedding $H_{0}^{1}(\Omega) \hookrightarrow L^{s}(\Omega)$ is not compact if $s=2^{*}$.

Now, fix $r>0$ and put

$$
\begin{gathered}
\lambda_{r}^{*}=\frac{r}{\left(\frac{\mu}{q} c_{q}^{q}(2 r)^{q / 2}+\frac{(2 r)^{2^{*} / 2}}{2^{*}} c_{2^{*}}^{2^{*}}\right)}, \quad \tilde{\lambda}_{r}=\frac{1}{c_{2^{*}}^{2^{*}}(2 r N)^{\frac{2}{N-2}}}, \\
\bar{\lambda}_{r}=\min \left\{\lambda_{r}^{*}, \tilde{\lambda}_{r}\right\},
\end{gathered}
$$

where $c_{q}, c_{2^{*}}$ are given by (4.2) and (4.1).

Now, we establish the following result.

Lemma 4.1. Let $\Phi$ and $\Psi$ be the functional defined as above and fix $r>0$. Then, for each $\lambda \in] 0, \bar{\lambda}_{r}\left[\right.$ the functional $I_{\lambda}=\Phi-\lambda \Psi$ satisfies the $(P S)^{[r]}-$ condition.

Proof. Fix $\lambda$ as in the conclusion and let $\left\{u_{n}\right\} \subseteq X$ be a sequence such that

$(\alpha)\left\{I_{\lambda}\left(u_{n}\right)\right\}$ is bounded,

( $\beta) \lim _{n \rightarrow+\infty}\left\|I_{\lambda}^{\prime}\left(u_{n}\right)\right\|_{X^{*}}=0$

$(\gamma) \Phi\left(u_{n}\right)<r \forall n \in \mathbb{N}$.

In particular, from $\Phi\left(u_{n}\right)<r \forall n \in \mathbb{N}$ we obtain that $\left\{u_{n}\right\}$ is bounded in $X$. So, going to a subsequence if necessary, we can assume $u_{n} \rightarrow u_{0}$ in $X, u_{n} \rightarrow u_{0}$ in $L^{q}(\Omega), u_{n} \rightarrow u_{0}$ a.e. on $\Omega$ and, taking $(\alpha)$ into account, $\lim _{n \rightarrow \infty} I_{\lambda}\left(u_{n}\right)=c$. Moreover, $\left\{u_{n}\right\}$ is bounded in $L^{2^{*}}(\Omega)$.

First step. We prove that $u_{0}$ is a weak solution of problem $\left(D_{\lambda}\right)$.

Since $\left\{u_{n}\right\}$ is bounded in $L^{2^{*}}(\Omega)$, it follows that $\left\{h\left(u_{n}\right)\right\}$ is bounded in $L^{\frac{2^{*}}{2^{*}-1}}(\Omega)$. Indeed, one has $\int_{\Omega}\left|h\left(u_{n}\right)\right|^{\frac{2^{*}}{2^{*}-1}} d x \leq \int_{\Omega}\left|u_{n}\right|^{2^{*}} d x$. Therefore, it follows that $h\left(u_{n}\right) \rightarrow h\left(u_{0}\right)$ in $L^{\frac{2^{*}}{2^{*}-1}}(\Omega)$. In fact, since $h$ is continuous and $u_{n} \rightarrow u_{0}$ a.e. 
$x \in \Omega$, we obtain $h\left(u_{n}\right) \rightarrow h\left(u_{0}\right)$ a.e. $x \in \Omega$, and that, together with the boundedness of $\left\{h\left(u_{n}\right)\right\}$ in $L^{\frac{2^{*}}{2^{*}-1}}(\Omega)$, ensures the weak convergence of $h\left(u_{n}\right)$ to $h\left(u_{0}\right)$ in $L^{\frac{2^{*}}{2^{*}-1}}(\Omega)$ (see [8, Remark (iii)]).

Moreover, since $u_{n} \rightarrow u_{0}$ in $L^{q}(\Omega)$, taking into account [14, Theorem A.2], one has that $g\left(u_{n}\right) \rightarrow g\left(u_{0}\right)$ in $L^{\frac{q}{q-1}}(\Omega)$. So, in particular, $g\left(u_{n}\right) \rightarrow g\left(u_{0}\right)$ in $L^{\frac{q}{q-1}}(\Omega)$.

Due to what was seen before, that is, $u_{n} \rightarrow u_{0}$ in $X, h\left(u_{n}\right) \rightarrow h\left(u_{0}\right)$ in $L^{\frac{2^{*}}{2^{*}-1}}(\Omega)$ and $g\left(u_{n}\right) \rightarrow g\left(u_{0}\right)$ in $L^{\frac{q}{q-1}}(\Omega)$, one has

$\lim _{n \rightarrow+\infty}\left(\int_{\Omega} \nabla u_{n}(x) \nabla v(x) d x-\lambda \int_{\Omega} h\left(u_{n}(x)\right) v(x) d x-\lambda \mu \int_{\Omega} g\left(u_{n}(x)\right) v(x) d x\right)=$ $\int_{\Omega} \nabla u_{0}(x) \nabla v(x) d x-\lambda \int_{\Omega} h\left(u_{0}(x)\right) v(x) d x-\lambda \mu \int_{\Omega} g\left(u_{0}(x)\right) v(x) d x$ for all $v \in$ $H_{0}^{1}(\Omega)$. Therefore, owing to $(\beta)$ we obtain that $0=\int_{\Omega} \nabla u_{0}(x) \nabla v(x) d x-$ $\lambda \int_{\Omega} h\left(u_{0}(x)\right) v(x) d x-\lambda \mu \int_{\Omega} g\left(u_{0}(x)\right) v(x) d x$ for all $v \in H_{0}^{1}(\Omega)$, that is, $u_{0}$ is a weak solution of $\left(D_{\lambda}\right)$.

Second step. We prove that

$$
I_{\lambda}\left(u_{0}\right)>-r .
$$

In fact, $\Psi(u)=\int_{\Omega} F(u(x)) d x \leq \frac{\mu}{q}\|u\|_{L^{q}(\Omega)}^{q}+\frac{1}{2^{*}}\|u\|_{L^{2^{*}}(\Omega)}^{2^{*}} \leq \frac{\mu}{q} c_{q}^{q}\|u\|^{q}+$ $\frac{1}{2^{*}} c_{2^{*}}^{2^{*}}\|u\|^{2^{*}}$. Hence,

$$
\Psi(u) \leq \frac{\mu}{q} c_{q}^{q}\|u\|^{q}+\frac{1}{2^{*}} c_{2^{*}}^{2^{*}}\|u\|^{2^{*}}, \quad \forall u \in X .
$$

Therefore, for all $u \in X$ such that $\|u\| \leq(2 r)^{1 / 2}$ one has $I_{\lambda}(u)=\Phi(u)-$ $\lambda \Psi(u) \geq \frac{\|u\|^{2}}{2}-\lambda\left(\frac{\mu}{q} c_{q}^{q}\|u\|^{q}+\frac{1}{2^{*}} c_{2^{*}}^{2^{*}}\|u\|^{2^{*}}\right) \geq-\lambda\left(\frac{\mu}{q} c_{q}^{q}(2 r)^{q / 2}+\frac{1}{2^{*}} c_{2^{*}}^{2^{*}}(2 r)^{2^{*} / 2}\right)=$ $-\lambda \frac{r}{\lambda_{r}^{*}}>-r$. So, taking into account $(\gamma)$ and that $\Phi$ is sequentially weakly lower semicontinuous, we have $\left\|u_{0}\right\| \leq \liminf _{n \rightarrow \infty}\left\|u_{n}\right\| \leq \sqrt{2 r}$ and, hence, $I_{\lambda}\left(u_{0}\right)>-r$.

Third step. Put $v_{n}=u_{n}-u_{0}$. We prove that one has

$$
c=\Phi\left(u_{0}\right)-\lambda \Psi\left(u_{0}\right)+\lim _{n \rightarrow \infty}\left(\frac{1}{2}\left\|v_{n}\right\|^{2}-\lambda \int_{\Omega} H\left(v_{n}\right) d x\right) .
$$

In fact, one has $\left\|u_{n}\right\|^{2}=\left\|v_{n}+u_{0}\right\|^{2}=\left\|v_{n}\right\|^{2}+\left\|u_{0}\right\|^{2}+2<v_{n}, u_{0}>$, so, it follows that

$$
\left\|u_{n}\right\|^{2}=\left\|v_{n}\right\|^{2}+\left\|u_{0}\right\|^{2}+o(1) .
$$


Moreover, the Brezis-Lieb Lemma (see [8, Theorem 1]) leads to

$$
\int_{\Omega} H\left(u_{n}\right) d x=\int_{\Omega} H\left(v_{n}\right) d x+\int_{\Omega} H\left(u_{0}\right) d x+o(1)
$$

(see also $[14, \mathrm{p} .33])$.

Finally, since $u \rightarrow \int_{\Omega} G(u) d x$ is locally Lipschitz in $L^{q}(\Omega)$ (see, for example, [10, Theorem 7.2.1]) and $u_{n} \rightarrow u_{0}$ in $L^{q}(\Omega)$, one has

$$
\int_{\Omega} G\left(u_{n}\right) d x=\int_{\Omega} G\left(u_{0}\right) d x+o(1)
$$

Hence, by starting from $c=\lim _{n \rightarrow \infty}\left(\Phi\left(u_{n}\right)-\lambda \Psi\left(u_{n}\right)\right)$, one has $c=\Phi\left(u_{n}\right)-\lambda \Psi\left(u_{n}\right)+o(1)=\frac{1}{2}\left\|u_{n}\right\|^{2}-\lambda \int_{\Omega} H\left(u_{n}\right) d x-\lambda \mu \int_{\Omega} G\left(u_{n}\right) d x+o(1)=$ $\frac{1}{2}\left\|v_{n}\right\|^{2}+\frac{1}{2}\left\|u_{0}\right\|^{2}-\lambda \int_{\Omega} H\left(v_{n}\right) d x-\lambda \int_{\Omega} H\left(u_{0}\right) d x-\lambda \mu \int_{\Omega} G\left(u_{0}\right) d x+o(1)=$ $\Phi\left(u_{0}\right)-\lambda \Psi\left(u_{0}\right)+\frac{1}{2}\left\|v_{n}\right\|^{2}-\lambda \int_{\Omega} H\left(v_{n}\right) d x+o(1)$. Hence, (B) is proved.

Fourth step. We prove the following

$$
\lim _{n \rightarrow \infty}\left(\left\|v_{n}\right\|^{2}-\lambda 2^{*} \int_{\Omega} H\left(v_{n}\right) d x\right)=0
$$

In fact, from $(\beta)$ we have $\lim _{n \rightarrow \infty} I^{\prime}\left(u_{n}\right)\left(u_{n}\right)=0$. So,

$$
\int_{\Omega} \nabla u_{n} \nabla u_{n} d x-\lambda \int_{\Omega}\left|u_{n}\right|^{2^{*}-1} u_{n} d x-\lambda \mu \int_{\Omega} g\left(u_{n}\right) u_{n} d x=o(1),
$$

for which, taking into account that $h\left(u_{n}\right) u_{n}=2^{*} H\left(u_{n}\right)$, one has $\left\|u_{n}\right\|^{2}-$ $\lambda 2^{*} \int_{\Omega} H\left(u_{n}\right) d x-\lambda \mu \int_{\Omega} g\left(u_{n}\right) u_{n} d x=o(1)$. Therefore, as seen in the proof of (B) and taking into account that $\int_{\Omega} g\left(u_{n}\right) u_{n} d x=\int_{\Omega} g\left(u_{0}\right) u_{0} d x+o(1)$ owing to the fact that $g\left(u_{n}\right) \rightarrow g\left(u_{0}\right)$ in $L^{\frac{q}{q-1}}(\Omega)$ (see the first step) and $u_{n} \rightarrow$ $u_{0}$ in $L^{q}(\Omega)$, one has $\left\|v_{n}\right\|^{2}+\left\|u_{0}\right\|^{2}-\lambda 2^{*} \int_{\Omega} H\left(v_{n}\right) d x-\lambda 2^{*} \int_{\Omega} H\left(u_{0}\right) d x-$ $\lambda \mu \int_{\Omega} g\left(u_{0}\right) u_{0} d x=o(1)$, that is,

$$
\left\|v_{n}\right\|^{2}-\lambda 2^{*} \int_{\Omega} H\left(v_{n}\right) d x=-\left\|u_{0}\right\|^{2}+\lambda 2^{*} \int_{\Omega} H\left(u_{0}\right) d x+\lambda \mu \int_{\Omega} g\left(u_{0}\right) u_{0} d x+o(1) .
$$

Since $u_{0}$ is a weak solution of $\left(D_{\lambda}\right)$, one has

$$
\left\|u_{0}\right\|^{2}-\lambda 2^{*} \int_{\Omega} H\left(u_{0}\right) d x-\lambda \mu \int_{\Omega} g\left(u_{0}\right) u_{0} d x=0 .
$$


Therefore,

$$
\left\|v_{n}\right\|^{2}-\lambda 2^{*} \int_{\Omega} H\left(v_{n}\right) d x=o(1)
$$

that is, (C) is proved.

Conclusion. Finally, we observe that $\left\|v_{n}\right\|^{2}$ is bounded in $\mathbb{R}$ since $u_{n}$ is bounded in $\mathrm{X}$. Thus, there is a subsequence, called again $\left\|v_{n}\right\|^{2}$, which converges to $b \in \mathbb{R}$. Hence,

$$
\lim _{n \rightarrow \infty}\left\|v_{n}\right\|^{2}=b .
$$

If $b=0$ we have proved the lemma. In fact, we have that $\lim _{n \rightarrow \infty}\left\|u_{n}-u_{0}\right\|=$ 0 , that is, $u_{n}$ strongly converges to $u_{0}$ in $X$. So, arguing by contradiction, we assume that $b \neq 0$. From (C) we obtain $\lim _{n \rightarrow \infty} \lambda 2^{*} \int_{\Omega} H\left(v_{n}\right) d x=b$. Now, taking into account that $\left\|v_{n}\right\|_{L^{2^{*}}(\Omega)} \leq c_{2^{*}}\left\|v_{n}\right\|$, for which $2^{*} \int_{\Omega} H\left(v_{n}\right) d x \leq$ $\int_{\Omega}\left|v_{n}\right|^{2^{*}} d x \leq c_{2^{*}}^{2^{*}}\left\|v_{n}\right\|^{2^{*}}$, and passing to the limit, one has $\frac{b}{\lambda} \leq c_{2^{*}}^{2^{*}} b^{2^{*} / 2}$ and then, since $b \neq 0$, one has

$$
b \geq\left(\frac{1}{\lambda}\right)^{\frac{N-2}{2}}\left(\frac{1}{c_{2^{*}}}\right)^{N} .
$$

Now, taking (A) into account, from (B) we have $c=\Phi\left(u_{0}\right)-\lambda \Psi\left(u_{0}\right)+\frac{1}{2} b-$ $\frac{1}{2^{*}} b>-r+\left(\frac{1}{2}-\frac{1}{2^{*}}\right) b=-r+\frac{1}{N} b$, that is

$$
c>-r+\frac{1}{N} b .
$$

On the other hand, since $F(\xi) \geq 0$ for all $\xi \in \mathbb{R}$, one has $\Phi\left(u_{n}\right)-\lambda \Psi\left(u_{n}\right)<r$ for all $n \in \mathbb{N}$. Hence, we have

$$
c \leq r .
$$

Thus, $-r+\frac{1}{N} b<c \leq r$. It follows that $\frac{1}{N} b<2 r$, that is,

$$
b<2 r N \text {. }
$$

Therefore, one has $\left(\frac{1}{\lambda}\right)^{\frac{N-2}{2}}\left(\frac{1}{c_{2^{*}}}\right)^{N} \leq b<2 r N$, so, it follows that $\frac{1}{\lambda}<$ $\left(2 r N c_{2^{*}}^{N}\right)^{\frac{2}{N-2}}$. Hence, one has

$$
\lambda>\frac{1}{(2 r N)^{\frac{2}{N-2}}} \frac{1}{c_{2^{*}}^{2 *}}=\tilde{\lambda}_{r}
$$


and this is a contradiction.

Remark 4.1. We observe that Lemma 4.1 is different from [6, Lemma 3.1] because of the role played here by the parameter $\mu$.

We also observe that the proof of the previous lemma is inspired by the classical one (see [14, Lemma 1.44]). The difference between the two proofs is, in particular, in the point (A) and, hence, in the conclusion.

Now, we can give the main result of this paper.

Theorem 4.1. Fix $q \in] 1,2\left[\right.$. Then, there exists $\mu^{*}>0$, where

$$
\mu^{*}=\left(\frac{q}{c_{q}^{q}} \frac{1}{2^{\frac{q+2}{2}}}\right)\left(\min \left\{\left(\frac{2^{*}}{2^{\frac{2^{*}+2}{2}} c_{2^{*}}^{2^{*}}}\right)^{\frac{2}{2^{*}-2}} ; \frac{1}{3 N}\left(\frac{1}{c_{2^{*}}^{2^{*}}}\right)^{\frac{N-2}{2}}\right\}\right)^{\frac{2-q}{2}}
$$

and $c_{q}, c_{2^{*}}$ are given by (4.2) and (4.1), such that for each $\left.\mu \in\right] 0, \mu^{*}[$ problem

$$
\left\{\begin{array}{l}
-\Delta u=u^{2^{*}-1}+\mu u^{q-1} \quad \text { in } \Omega, \\
\left.u\right|_{\partial \Omega}=0
\end{array}\right.
$$

admits at least one positive weak solution $u_{\mu}$ such that

$$
\left\|u_{\mu}\right\|<\left(\frac{2^{*}}{c_{2^{*}}^{2^{*}}}\right)^{\frac{1}{2^{*}-2}}
$$

Moreover, the mapping

$$
\mu \rightarrow \frac{1}{2} \int_{\Omega}\left|\nabla u_{\mu}\right|^{2} d x-\int_{\Omega} \frac{1}{2^{*}}\left|u_{\mu}\right|^{2^{*}} d x-\mu \int_{\Omega} \frac{1}{q}\left|u_{\mu}\right|^{q} d x
$$

is negative and strictly decreasing in $] 0, \mu^{*}[$.

Proof. Our aim is to apply Theorem 3.3 and to this end we use the usual setting. Precisely, put $X=H_{0}^{1}(\Omega)$ endowed with the norm $\|u\|=\left(\int_{\Omega}|\nabla u(x)|^{2} d x\right)^{\frac{1}{2}}$ and $\Phi(u)=\frac{\|u\|^{2}}{2}, \Psi(u)=\int_{\Omega} F(u(x)) d x$ for all $u \in X$, where $F(\xi)=\int_{0}^{\xi} f(t) d t$ for every $\xi \in \mathbb{R}$ and $f(t)=h(t)+\mu g(t)$ for all $t \in \mathbb{R}, f(t)=t^{2^{*}-1}, g(t)=t^{q-1}$ for all $t \geq 0$ and $f(t)=g(t)=0$ for all $t<0$.

Now, put

$$
r=\min \left\{\left(\frac{2^{*}}{2^{\frac{2^{*}+2}{2}} c_{2^{*}}^{2^{*}}}\right)^{\frac{2}{2^{*}-2}} ; \frac{1}{3 N}\left(\frac{1}{c_{2^{*}}^{2^{*}}}\right)^{\frac{N-2}{2}}\right\}
$$


and

$$
\mu^{*}=\left(\frac{q}{c_{q}^{q}} \frac{1}{2^{\frac{q+2}{2}}}\right) r^{\frac{2-q}{2}} .
$$

Fix $0<\mu<\mu^{*}$, and one has $\bar{\lambda}_{r}>1$. Indeed,

$$
\begin{aligned}
\tilde{\lambda}_{r}=\frac{1}{c_{2^{*}}^{2^{*}}(2 r N)^{\frac{2}{N-2}}} \geq \frac{1}{c_{2^{*}}^{2^{*}}(2 N)^{\frac{2}{N-2}}\left[\frac { 1 } { 3 N } \left(\frac{1}{\left.\left.c_{2^{*}}^{2^{*}}\right)^{\frac{N-2}{2}}\right]^{\frac{2}{N-2}}}=\left(\frac{3}{2}\right)^{\frac{2}{N-2}}>1\right.\right. \text { and }} \\
\lambda_{r}^{*}=\frac{1}{\frac{\mu}{q} c_{q}^{q} 2^{q / 2} r^{\frac{q-2}{2}}+\frac{2^{2^{*} / 2}}{2^{*}} c_{2^{*}}^{2^{*}} r^{\frac{2^{*}-2}{2}}} \\
\geq \frac{\frac{\mu}{q} c_{q}^{q} 2^{q / 2} r^{\frac{q-2}{2}}+\frac{2^{2^{*} / 2}}{2^{*}} c_{2^{*}}^{2^{*}}\left[\left(\frac{2^{*}}{2^{\frac{2^{*}+2}{2}} c_{2^{*}}^{2^{*}}}\right)^{\frac{2}{2^{*}-2}}\right]^{\frac{2^{*}-2}{2}}}{\frac{1}{\mu^{*}} c_{q}^{q} 2^{q / 2} r^{\frac{q-2}{2}}+\frac{1}{2}} \\
>
\end{aligned}
$$

Therefore, from Lemma 4.1, the functional $I_{\lambda}=\Phi-\lambda \Psi$ satisfies the $(P S)^{[r]}{ }_{-}$ condition for all $\lambda \in] 0 . \bar{\lambda}_{r}[$.

Now, fix $\lambda<\bar{\lambda}_{r}$. We claim that there is $v_{0} \in X$, with $0<\Phi\left(v_{0}\right)<r$, such that

$$
\frac{\sup _{u \in \Phi^{-1}(]-\infty, r[)} \Psi(u)}{r}<\frac{\Psi\left(v_{0}\right)}{\Phi\left(v_{0}\right)} .
$$

To this end, taking into account that $\|u\|_{L^{s}(\Omega)} \leq c_{s}\|u\|, \quad u \in H_{0}^{1}(\Omega)$, one has

$$
\begin{gathered}
\sup _{\frac{u \in \Phi^{-1}(]-\infty, r[)}{r} \Psi(u)} \leq \frac{\sup _{u \in \Phi^{-1}(]-\infty, r[)} \int_{\Omega} F(u(x)) d x}{r} \leq \\
\leq \frac{\sup _{u \in \Phi^{-1}(]-\infty, r[)}\left(\frac{\mu}{q}\|u\|_{L^{q}(\Omega)}^{q}+\frac{1}{2^{*}}\|u\|_{L^{2^{*}}(\Omega)}^{2^{*}}\right)}{r} \leq \\
\leq \frac{\sup _{u \in \Phi^{-1}(]-\infty, r[)}\left(\frac{\mu}{q} c_{q}^{q}\|u\|^{q}+\frac{1}{2^{*}} c_{2^{*}}^{2^{*}}\|u\|^{2^{*}}\right)}{r} \leq \frac{\left.\left(\frac{\mu}{q} c_{q}^{q}(2 r)\right)^{q}+\frac{1}{2^{*}} c_{2^{*}}^{2^{*}}(2 r)^{2^{*}}\right)}{r} .
\end{gathered}
$$


Hence, one has

$$
\frac{\sup _{u \in \Phi^{-1}(]-\infty, r[)} \Psi(u)}{r} \leq \frac{1}{\lambda_{r}^{*}}<\frac{1}{\lambda}
$$

Next, fix

$$
R=\sup _{x \in \Omega} d(x, \partial \Omega),
$$

and let $x_{0} \in \Omega$ such that $B\left(x_{0}, R\right) \subseteq \Omega$. Moreover, put

$$
v_{\delta}(x):= \begin{cases}0 & \text { if } x \in \Omega \backslash B\left(x_{0}, R\right) \\ \frac{2 \delta}{R}\left(R-\left|x-x_{0}\right|\right) & \text { if } x \in B\left(x_{0}, R\right) \backslash B\left(x_{0}, R / 2\right) \\ \delta & \text { if } x \in B\left(x_{0}, R / 2\right) .\end{cases}
$$

Clearly, one has that $v_{\delta} \in X$ and

$$
\begin{gathered}
\Phi\left(v_{\delta}\right)=\frac{1}{2} \int_{\Omega}\left|\nabla v_{\delta}(x)\right|^{2} d x= \\
=\frac{1}{2} \int_{B\left(x_{0}, R\right) \backslash B\left(x_{0}, R / 2\right)} \frac{(2 \delta)^{2}}{R^{2}} d x=\frac{1}{2} \frac{(2 \delta)^{2}}{R^{2}}\left(\operatorname{meas}\left(B\left(x_{0}, R\right)\right)-\operatorname{meas}\left(B\left(x_{0}, R / 2\right)\right)\right)= \\
=\frac{1}{2} \frac{(2 \delta)^{2}}{R^{2}} \frac{\pi^{N / 2}}{\Gamma(1+N / 2)}\left(R^{N}-(R / 2)^{N}\right),
\end{gathered}
$$

where $\Gamma$ is the Euler function. Moreover, one has $\Psi\left(v_{\delta}\right)=\int_{\Omega} F\left(v_{\delta}(x)\right) d x \geq$ $\int_{B\left(x_{0}, R / 2\right)} F(\delta) d x \geq F(\delta) \frac{\pi^{N / 2}}{\Gamma(1+N / 2)} \frac{R^{N}}{2^{N}}$ and, hence,

$$
\frac{\Psi\left(v_{\delta}\right)}{\Phi\left(v_{\delta}\right)} \geq \frac{R^{2}}{2\left(2^{N}-1\right)} \frac{F(\delta)}{\delta^{2}}
$$

From $\limsup _{t \rightarrow 0^{+}} \frac{G(t)}{t^{2}}=+\infty$ it follows that $\limsup _{t \rightarrow 0^{+}} \frac{F(t)}{t^{2}}=+\infty$. So, taking into account that $\Phi\left(v_{\delta}\right)=\frac{1}{2} \frac{(2 \delta)^{2}}{R^{2}} \frac{\pi^{N / 2}}{\Gamma(1+N / 2)}\left(R^{N}-(R / 2)^{N}\right)$, there is a $\bar{\delta}>0$ such that

$$
\frac{R^{2}}{2\left(2^{N}-1\right)} \frac{F(\bar{\delta})}{\bar{\delta}^{2}}>\frac{1}{\lambda}
$$

and $\Phi\left(v_{\bar{\delta}}\right)<r$. 
Therefore, $\frac{\sup _{u \in \Phi^{-1}(]-\infty, r[)} \Psi(u)}{r}<\frac{1}{\lambda}<\frac{R^{2}}{2\left(2^{N}-1\right)} \frac{F(\bar{\delta})}{\bar{\delta}^{2}} \leq \frac{\Psi\left(v_{\bar{\delta}}\right)}{\Phi\left(v_{\bar{\delta}}\right)}$ with $0<$ $\Phi\left(v_{\bar{\delta}}\right)<r$. Hence, our claim is proved.

Finally, from Theorem 3.3 the functional $\Phi-\lambda \Psi$ admits a critical point $u_{\lambda, \mu}$ such that $\frac{1}{2}\left\|u_{\lambda, \mu}\right\|^{2}>0$, which is a positive weak solution for problem $\left(D_{\lambda}\right)$. In particular, by choosing $\lambda=1$, a positive weak solution $u_{\mu}$ for problem $\left(D_{\mu}\right)$ is obtained. Moreover, one has $\frac{1}{2}\left\|u_{\mu}\right\|^{2}<r$, from which $\frac{1}{2}\left\|u_{\mu}\right\|^{2}<$ $\left(\frac{2^{*}}{2^{\frac{2^{*}+2}{2}} c_{2^{*}}^{2^{*}}}\right)^{\frac{2}{2^{*}-2}}$, that is, $\left\|u_{\mu}\right\|<\left(\frac{2^{*}}{c_{2^{*}}^{2^{*}}}\right)^{\frac{1}{2^{*}-2}}$.

Now, since $u_{\mu}$ is a global minimum for $I_{1}$ in $\Phi^{-1}(] 0, r[)$ again from Theorem 3.3 , and $v_{\bar{\delta}} \in \Phi^{-1}(] 0, r[)$, one has $I_{1}\left(u_{\mu}\right) \leq I_{1}\left(v_{\bar{\delta}}\right)$. So, taking into account that $\frac{\Psi\left(v_{\bar{\delta}}\right)}{\Phi\left(v_{\bar{\delta}}\right)}>\frac{1}{\lambda}=1$ as seen before, one has $I_{1}\left(u_{\mu}\right) \leq I_{1}\left(v_{\bar{\delta}}\right)<0$. Next, fix $0<\mu_{1}<\mu_{2}$. One has

$I_{1}\left(u_{\mu_{1}}\right)=\min _{u \in \Phi^{-1}(] 0, r[)}\left(\frac{1}{2} \int_{\Omega}|\nabla u|^{2} d x-\int_{\Omega} \frac{1}{2^{*}}|u|^{2^{*}} d x-\mu_{1} \int_{\Omega} \frac{1}{q}|u|^{q} d x\right)>$ $\min _{u \in \Phi^{-1}(] 0, r[)}\left(\frac{1}{2} \int_{\Omega}|\nabla u|^{2} d x-\int_{\Omega} \frac{1}{2^{*}}|u|^{2^{*}} d x-\mu_{2} \int_{\Omega} \frac{1}{q}|u|^{q} d x\right)=I_{1}\left(u_{\mu_{2}}\right)$ and the conclusion is achieved.

Remark 4.2. The existence of at least one solution to problem $\left(D_{\mu}\right)$ was previously proved in [1] by using topological methods, that is, the method of sub- and super-solution. The proof of Theorem 4.1 was obtained by variational methods, that is, via a local minimum result as established in Theorem 3.3. We also observe that, [1, Theorem 2.1] establishes, in particular, the existence of $\Lambda>0$ such that problem $\left(D_{\mu}\right)$ admits a solution for each $\left.\left.\mu \in\right] 0, \Lambda\right]$ and no solution for $\mu>\Lambda$. However, no numerical estimate of $\Lambda$ was pointed out in [1]. Here, we point out that from Theorem 4.1 one has

$$
\Lambda \geq \mu^{*} .
$$

It follows that, from Theorem 4.1, the existence of solutions for $\left(D_{\mu}\right)$ is obtained for suitable numerical values of $\mu$ (see Example 4.1).

Remark 4.3. [1, Theorem 2.3] ensures the existence of a second solution for $\left(D_{\mu}\right)$ for all $\left.\mu \in\right] 0, \Lambda$, by applying a version of the mountain pass theorem. In order to apply the mountain pass theorem, the authors prove, by a careful examination, that the solution obtained by topological methods is actually a local minimum of a suitable functional. We observe that the solution obtained 
in Theorem 4.1 is directly a local minimum for such a functional. So, to obtain the second solution is enough to apply the mountain pass theorem arguing as in part of the proof of [1, Theorem 2.3]. The details are in the proof of Theorem 4.2 .

Finally, as a consequence of Theorem 4.2, we obtain the following existence result of two solutions, where an estimate of parameters is pointed out.

Theorem 4.2. Fix $q \in] 1,2\left[\right.$. Then, there exists $\mu^{*}>0$, where

$$
\mu^{*}=\left(\frac{q}{c_{q}^{q}} \frac{1}{2^{\frac{q+2}{2}}}\right)\left(\min \left\{\left(\frac{2^{*}}{2^{\frac{2^{*}+2}{2}} c_{2^{*}}^{2^{*}}}\right)^{\frac{2}{2^{*}-2}} ; \frac{1}{3 N}\left(\frac{1}{c_{2^{*}}^{2^{*}}}\right)^{\frac{N-2}{2}}\right\}\right)^{\frac{2-q}{2}}
$$

and $c_{q}, c_{2 *}$ are given by (4.2) and (4.1), such that for each $\left.\mu \in\right] 0, \mu^{*}[$ problem $\left(D_{\mu}\right)$ admits at least two positive weak solutions $u_{\mu}$ and $w_{\mu}$ such that $\left\|u_{\mu}\right\|<$ $\left(\frac{2^{*}}{c_{2^{*}}^{2^{*}}}\right)^{\frac{1}{2^{*}-2}}$ and $w_{\mu}>u_{\mu}$.

Proof. Fix $\mu \in] 0, \mu^{*}[$. From Theorem 4.1 there exists a positive weak solution $u_{\mu}$ of $\left(D_{\mu}\right)$ such that $u_{\mu}$ is a local minimum for the functional $I(u)=\Phi(u)-$ $\Psi(u)=\frac{\|u\|^{2}}{2}-\int_{\Omega} F(u(x)) d x$, where $F$ is the primitive of $f(t)=t^{2^{*}-1}+\mu t^{q-1}$ if $t \geq 0$ and $f(t)=0$ if $t<0$. Now consider the problem

$$
\left\{\begin{aligned}
-\Delta v & =\left(u_{\mu}+v\right)^{2^{*}-1}-u_{\mu}^{2^{*}-1}+\mu\left(u_{\mu}+v\right)^{q-1}-\mu u_{\mu}^{q-1} \quad \text { in } \Omega, \\
\left.v\right|_{\partial \Omega} & =0 .
\end{aligned}\right.
$$

Clearly, if $v_{\mu}$ is a positive weak solution to $\left(P_{\mu}\right)$, then $w_{\mu}=u_{\mu}+v_{\mu}$ is a weak solution of $\left(D_{\mu}\right)$ such that $w_{\mu}>u_{\mu}>0$. So, our aim is to prove that $\left(P_{\mu}\right)$ admits at least one positive weak solution. To this end, consider the functional $J$ defined as $J(v)=\frac{\|v\|^{2}}{2}-\int_{\Omega} L(x, v(x)) d x$, where $L(x, \xi)=\int_{0}^{\xi} l(x, t) d t$ and $l(x, t)=\left(u_{\mu}(x)+t\right)^{2^{*}-1}-\left[u_{\mu}(x)\right]^{2^{*}-1}+\mu\left(u_{\mu}(x)+t\right)^{q-1}-\mu\left[u_{\mu}(x)\right]^{q-1}$ if $t \geq 0$, $l(x, t)=0$ if $t<0$. Clearly, non-zero critical points of $J$ are positive weak solution of $\left(P_{\mu}\right)$. Now, we observe that 0 is a local minimum of $J$. Indeed, since $u_{\mu}$ is a local minimum of $I$, one has $I\left(u_{\mu}+v\right)-I\left(u_{\mu}\right) \geq 0$ for all $v \in X$ such that $\|v\|<\delta$ for some $\delta>0$. So, taking into account that

$$
J(v)=\frac{1}{2}\left\|v^{-}\right\|^{2}+I\left(u_{\mu}+v^{+}\right)-I\left(u_{\mu}\right) \geq 0
$$

for all $v \in X$ (see [1, pag.1 line 11]), one has $J(v) \geq 0$ for all $v \in X$ such that $\|v\|<\delta$ and our claim is proved. 
At this point arguing exactly as in [1], the functional $J$ admits a positive critical point $v_{\mu}$ for which $w_{\mu}=u_{\mu}+v_{\mu}$ is the second weak solution of $\left(P_{\mu}\right)$ and the proof is complete.

Example 4.1. Fix $N=3$ and let $\Omega=\left\{x \in \mathbb{R}^{3}:|x|<1\right\}$. Then, the problem

$$
\left\{\begin{aligned}
-\Delta u & =u^{5}+\frac{3}{8} \sqrt{u} \quad \text { in } \quad \Omega, \\
\left.u\right|_{\partial \Omega} & =0
\end{aligned}\right.
$$

admits at least two positive weak solutions $u_{\mu}$ and $w_{\mu}$ such that $\int_{\Omega}\left|\nabla u_{\mu}(x)\right|^{2} d x<$ $\frac{9 \pi^{2}}{2^{5 / 2}}$ and $w_{\mu}>u_{\mu}$. In fact, it is enough to apply Theorem 4.2 by choosing $q=\frac{3}{2}$ and taking into account that $c_{2^{*}}^{2^{*}}=\frac{2^{6}}{3^{3} \pi^{4}}, c_{q}^{q} \leq \frac{2^{5 / 2}}{3^{3 / 2} \pi^{1 / 4}}$ for which $\mu^{*} \geq \frac{9}{256}\left(\frac{\pi^{9}}{2}\right)^{1 / 4}>\frac{3}{8}$.

Remark 4.4. We observe that we cannot apply [1, Theorem 2.3] to Problem $(P)$ since no estimate of $\Lambda$ was pointed out there (see also Remark 4.2).

\section{Acknowledgments}

The first two authors have been partially supported by the Gruppo Nazionale per l'Analisi Matematica, la Probabilità e le loro Applicazioni (GNAMPA) of the Istituto Nazionale di Alta Matematica (INdAM).

\section{References}

[1] A. Ambrosetti, H. Brézis and G. Cerami, Combined effects of concave and convex nonlinearities in some elliptic problems, J. Funct. Anal. 122 (1994), 519-543.

[2] G. Bonanno, G. Barletta and D. O'Regan, A variational approach to multiplicity results for boundary-value problems on the real line, Proc. Roy. Soc. Edinburgh Sect. A, 145 (2015), 13-29.

[3] G. Bonanno, A critical point theorem via the Ekeland variational principle, Nonlinear Anal. 75 (2012), 2992-3007.

[4] G. Bonanno, Relations between the mountain pass theorem and local minima, Adv. Nonlinear Anal. 1 (2012), 205-220. 
[5] G. Bonanno and P. Candito, Non-differentiable functionals and applications to elliptic problems with discontinuous nonlinearities, J. Differential Equations 244 (2008), 3031-3059.

[6] G. Bonanno and G. D'Aguì, Critical nonlinearities for elliptic Dirichlet problems, Dynam. Systems and Appl. 22 (2013), 411-418.

[7] G. Bonanno and G. D'Aguì, A critical point theorem and existence results for a nonlinear boundary value problem, Nonlinear Anal. 72 (2010), 19771982.

[8] H. Brézis and E. Lieb, A relation between pointwise convergence of functions and convergence of functionals, Proc. Amer. Math. Soc. 88 (1983), 486-490.

[9] H. Brézis and L. Nirenberg, Positive solutions of nonlinear elliptic equations involving critical Sobolev exponent, Comm. Pure Appl. Math. 36 (1983), 437-477.

[10] J. Chabrowski, Variational methods for potential operator equations, de Gruyter, Berlin, 1997.

[11] S. I. Pohozaev, Eigenfunctions of the equation $\Delta u+\lambda f(u)=0$, Soviet Math. Doklady 6 (1965), 1408-1411.

[12] B. Ricceri, A general variational principle and some of its applications, J. Comput. Appl. Math. 113 (2000), 401-410.

[13] M. Struwe, Variational Methods, Springer-Verlag, Berlin, 1996.

[14] M. Willem, Minimax theorems, Birkhäuser, Berlin, 1996.

Gabriele Bonanno,

Department of Engineering,

University of Messina,

c/da Di Dio, Messina 98166 - Italy

Email: bonanno@unime.it

Giuseppina D'Aguì

Department of Engineering,

University of Messina,

c/da Di Dio, Messina 98166 - Italy

Email: gdagui@unime.it

Donal O'Regan,

School of Mathematics, Statistics and Applied Mathematics,

National University of Ireland,

Galway, Ireland

Email: donal.oregan@nuigalway.ie 